\title{
EL NUEVO PARADIGMA DE LOS DERECHOS HUMANOS EN MÉXICO Y LA IMPORTANCIA DE LA ESPECIFICIDAD DE LA INTERPRETACIÓN CONSTITUCIONAL
}

\author{
The new paradigm of human rights in México and the importance of the specificity of \\ constitutional interpretation
}

José Jesús SORIANO FLORES

\section{Sumario:}

1. A manera de Introducción. 2. El nuevo orden constitucional de los derechos humanos en México. 2.1. Temas Fundamentales de la Reforma Constitucional de 2011. 2.2. Hacia un nuevo paradigma constitucional de los derechos humanos. 3. Algunas notas sobre la especificidad de la interpretación constitucional. 3.1. Consideraciones introductorias. 3.2. Sobre la interpretación constitucional . 3.2.1. Hacia una conceptualización de la interpretación constitucional. 3.2.2. La especificidad de la interpretación constitucional. 4. Reflexiones Finales.

Resumen: La reforma constitucional en México del año 2011, ha dado lugar a un nuevo paradigma constitucional de los derechos humanos, del cual se establecen en el texto una serie de elementos que lo identifican. Por lo anterior, es necesario atender diversos aspectos, como el de la especificidad de la interpretación constitucional de los derechos humanos, tópico del cual se esgrimen algunas consideraciones respecto a su conceptualización y alcance.

Palabras Clave: Constitución, Derechos Humanos, Interpretación Constitucional.

Abstract: The constitutional reform in México in 2011 has led to a new constitutional paradigm of human rights. Therefore, it is necessary to address various important aspects of the reform, such as the specificity of the constitutional interpretation of human rights. This paper discusses some considerations about the new paradigm and extent and conceptualization of the specificity of the constitutional interpretation.

Keywords: Constitution, Human Rights, Constitutional Interpretation.

\footnotetext{
${ }^{1}$ Profesor Investigador de tiempo completo del Departamento de Derecho de la División de Derecho, Política y Gobierno de la Universidad de Guanajuato, México y Miembro Cuerpo Académico: Ciencia Penal, Estado de Derecho y Derechos Humanos.

Maestro en Derecho Constitucional por la Universidad Iberoamericana y el Instituto de Investigaciones Jurídicas de la UNAM, Licenciado en Derecho por la Universidad de Guanajuato y alumno del Programa de Doctorado Interinstitucional en Derecho de las Universidades Públicas de la Región Centro Occidente de ANUIES. Correo electrónico: jesussorianomx@yahoo.com.mx
} 


\section{A MANERA DE INTRODUCCIÓN}

La historia reciente de los derechos humanos, al menos desde la perspectiva estrictamente jurídica, ha mostrado diversos avances importantes que se han concretado en modificaciones a criterios jurisdiccionales, y por supuesto a textos normativos, incluyendo a las Constituciones.

Aunque el desarrollo teórico y normativo de "derechos", es precisamente uno de los puntos torales del derecho constitucional, y que incluso la Constitución mexicana vigente fue en su momento elogiada por su contenido garantista en materia de derechos sociales, la teoría de los derechos humanos ha desarrollado progresivamente nuevos conceptos y contenidos, fundamentalmente a partir del término de la segunda guerra mundial, cuando se establece la primera carta internacional de derechos, denominada "Declaración Universal de los Derechos Humanos" (1948).

Estos acontecimientos, trajeron como consecuencia entre otras cuestiones, primero que los derechos humanos se internacionalizaran mediante la firma y ratificación de tratados internacionales, así como con el surgimiento de sistemas específicos de protección; elementos que han provocado entre otras cosas, la incorporación de nuevos y mejores estándares a los sistemas jurídicos nacionales, que implican de manera sobresaliente, nuevas maneras de entender la especificidad de la interpretación constitucional.

En este orden de ideas, primeramente abordaremos las razones por las cuales afirmamos que nos encontramos ante un nuevo paradigma constitucional en la materia, para después comentar brevemente algunos elementos en torno a esta idea de especificidad de la interpretación constitucional de los derechos humanos.

\section{EL NUEVO ORDEN CONSTITUCIONAL DE LOS DERECHOS HUMANOS EN MÉXICO}

\subsection{TEMAS FUNDAMENTALES DE LA REFORMA CONSTITUCIONAL DE 2011}

En el mes de mayo de 2011, se aprobó una de las reformas constitucionales en materia de derechos humanos más importantes en México, la cual fue publicada en el diario oficial de la federación el 10 de junio de 2011, y que entre otros temas fundamentales, desarrolla los siguientes: ${ }^{2}$

- De las garantías a los derechos: la modificación de la denominación del primer capítulo de la Constitución.

- La referencia preeminente de los tratados internacionales.

- Incorporación expresa del principio pro persona o pro homine.

-El Estado, como principal sujeto obligado.

- Hacia un desarrollo más garantista del derecho a la no discriminación.

\footnotetext{
${ }^{2}$ Cfr. VIDAURRI ARÉCHIGA, Manuel, SORIANO FLORES José Jesús, "El Contenido de la Reforma Constitucional de 2011 en materia de Derechos Humanos: 10 Temas Fundamentales”, en Revista Penal, núm. 30, Universidad de Huelva, España, 2012.
} 
- Los derechos humanos en la educación pública.

- Un nuevo orden respecto de la restricción o suspensión de derechos.

- Elementos para el fortalecimiento de los organismos públicos de derechos humanos.

- La obligatoriedad de legislar sobre determinadas materias. ${ }^{3}$

Los derechos humanos en el constitucionalismo mexicano, han pasado por momentos emblemáticos, y como en la historia universal, estas prerrogativas generalmente han atravesado por un camino muchas veces accidentado, a pesar de que desde la Constitución de Cádiz de 1812, promulgada primero en España y posteriormente en la Nueva España, reconoció algunos derechos, cuando en su artículo 4 estableció que "la nación está obligada a conservar y proteger por leyes sabias la libertad civil, la propiedad, y los demás derechos legítimos de todos los individuos que la componen".

Pues bien, después de otros textos constitucionales en México, ${ }^{4}$ la Constitución federal vigente representó un avance claro en esta materia, situación que colocó a ese texto constitucional como un referente internacional, fundamentalmente, como se mencionó, en la elevación de los derechos sociales al rango constitucional. En este sentido, en 1917 la Constitución denominó a su primer capítulo: "De las Garantías Individuales", pues como se aprecia en el diario de los debates de aquellos tiempos, influyeron ideas como las de José N. Macías, quien apuntaba que "las constituciones no necesitan declarar cuáles son los derechos; necesitan garantizar de la manera más completa y más absoluta todas las manifestaciones de la libertad; por eso deben otorgarse las garantías individuales".5

El texto constitucional entonces, hasta antes de la reforma de 2011, incorporó un apartado específico con derechos individuales y colectivos, sin embargo, el desarrollo reciente de los derechos humanos, fundamentalmente a partir de la internacionalización de los mismos en la década de los cuarentas, ha planteado una visión mucho más completa, integral y progresista, en donde la acepción "derechos humanos", distinta a la de garantías individuales, derechos del hombre o derechos naturales por ejemplo, establece un discurso jurídico y político coherente, más garantista y democrático en el que por ejemplo, los instrumentos internacionales en la materia, se convierten en un referente obligado.

En este orden de ideas, la reforma de mérito, representa un avance sustancial en la asignatura que nos ocupa, pues no estamos ante cambios "formales", sino "sustanciales", que nos sitúan frente a un nuevo paradigma en la interpretación y aplicación de los derechos humanos, paradigma que nosotros abordaremos fundamentalmente en el tema de la hermenéutica jurídico constitucional de los derechos humanos.

\footnotetext{
${ }^{3}$ Ley sobre Reparación del Daño por Violaciones a Derechos Humanos, Ley a que se refiere el artículo 11 constitucional sobre el Asilo, Ley Reglamentaria del artículo 29 constitucional en materia de Suspensión del ejercicio de los Derechos y las Garantías, y la Ley Reglamentaria del artículo 33 constitucional, en materia de Expulsión de Extranjeros.

${ }^{4}$ Es importante hacer notar que la Constitución de 1857 incorporó la noción "Derechos del Hombre".

5 Diario de Debates, t. I, pp. 1048 y 1050, citado por LARA PONTE, Rodolfo, Los Derechos Sociales en el Cons-

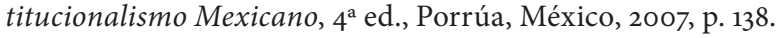




\subsection{HACIA UN NUEVO PARADIGMA CONSTITUCIONAL DE LOS DERECHOS HUMANOS}

Hay varias razones para afirmar que nos encontramos ante un nuevo paradigma constitucional; ${ }^{6}$ en este tenor, a continuación estableceremos, sin ser de ninguna manera exhaustivos, algunos elementos que sitúan sobre todo a las y los juzgadores en ese nuevo modelo.

La hermenéutica constitucional, tiene ahora mayores y más profundos alcances, pues en el marco de la reforma constitucional de 2011, por ejemplo la SCJN “deja de tener el monopolio en la interpretación de los derechos humanos, al existir una pluralidad de intérpretes del texto constitucional", 7 por lo que como lo señala Ferrer Mac Gregor, "el diálogo jurisprudencial se traslada al ámbito nacional entre la SCJN, el TRIFE, los tribunales superiores de las entidades federativas, y en general entre todos los jueces del país. (federales y locales)". ${ }^{8}$

Además, en este sentido, como lo ha mencionado la ministra Sánchez Cordero respecto a la hermenéutica de los derechos fundamentales, "mucho se ha escrito sobre la interpretación de las leyes ordinarias; sin embargo esas aportaciones no resultan del todo aplicables a la interpretación constitucional $y$, menos, a la de derechos fundamentales ya que ésta requiere de pautas hermenéuticas diversas", 9 donde principalmente las y los jueces juegan un papel determinante, cuyo protagonismo es entonces sin duda central desde la perspectiva de este orden constitucional, que posiciona a las y los administradores de justicia como los operadores del Estado que interpretan y aplican finalmente todo un modelo de política de Estado, sin importar la estructura jerárquica o competencial a la que pertenecen.

A pesar de que el artículo 133 constitucional ya establecía la obligatoriedad de los tratados internacionales como lo mencionamos con antelación, las y los jueces generalmente, en una práctica consuetudinaria avalada por el sistema jurídico, no solían incorporar en la interpretación y argumentación de sus resoluciones normas relativas a tratados internacionales de derechos humanos y mucho menos a otros instrumentos internacionales, ${ }^{10}$ no obstante, la reforma constitucional que hemos comentado, no solamente clarifica la obligatoriedad de dichos instrumentos, sino más aun, los eleva al rango constitucional, cuyos efectos de interpretación incluyen ahora incluso como criterios orientadores la jurisprudencia de la Corte

\footnotetext{
6 Se sugiere consultar: DE LA TORRE TORRES, Rosa María, "Ponencia: El nuevo paradigma de los derechos humanos en México", V Congreso Nacional de Derecho Constitucional, UNAM, México, D.F., Octubre de 2012.

7 FERRER MAC GREGOR, Eduardo, “Interpretación Conforme y Control Difuso de Convencionalidad: El nuevo paradigma para el Juez Mexicano”, en Estudios Constitucionales, Centro de Estudios Constitucionales de Chile Universidad de Talca, Año 9, No 2, 2011, pp. 531 - 622.

8 Ibidem.

9 SÁNCHEZ CORDERO, Olga, La interpretación de los derechos fundamentales por la Suprema Corte de Justicia de México, en línea: http://www.scjn.gob.mx/conocelacorte/ministra/o1junio-conferencia-derechosfundamentales.pdf, fecha de consulta: 1 de enero de 2013.

En el texto citado, la ministra, señala que "el problema sobre la determinación de la técnica idónea para la interpretación de los Derechos Fundamentales, representa en la actualidad un tema que pertenece, no sólo al ámbito de lo académico, sino también una cuestión con profundas implicaciones prácticas que, según mi experiencia, llegan a ser muy frecuentes en materia de justicia constitucional; y a pesar de ello, la bibliografía especializada, tanto nacional como extranjera, es escasa".

10 Tales como declaraciones internacionales, jurisprudencia de tribunales internacionales, y observaciones generales de algunos de los pactos de Naciones Unidas.
} 
Interamericana de derechos humanos, como se afirmó en el expediente varios 912/2010, derivado del caso Radilla Pacheco resuelto por la Suprema Corte de Justicia de la Nación.

La pluralidad de intérpretes del texto constitucional a la que nos referimos, entonces, por ejemplo obliga a la totalidad de las y los juzgadores a revisar en la construcción de una sentencia cuyo objeto de estudio sea el tipo penal de "tortura", además de las disposiciones relativas y aplicables del código penal, la Convención interamericana para prevenir y sancionar la tortura del sistema interamericano, y la Convención contra la tortura y otros tratos o penas crueles, inhumanos o degradantes del sistema universal, atendiendo a la "interpretación conforme", que textualmente refiere el texto constitucional.

Otro tópico de primer orden que nos sitúa ante el paradigma constitucional en comento, es precisamente la incorporación expresa de diversos principios de interpretación constitucional, como el principio "pro persona"11 cuando el texto refiere en el artículo primero que "las normas relativas a los derechos humanos se interpretarán de conformidad con esta Constitución y con los tratados internacionales de la materia favoreciendo en todo tiempo a las personas la protección más amplia"12. En este sentido, la tesis jurisprudencial 107/2012, denominada "Principio Pro persona. Criterio de selección de la norma de derecho fundamental aplicable”, establece que de conformidad con el artículo constitucional, existen dos fuentes primigenias, por un lado los derechos reconocidos en la propia Constitución; y por otro, los establecidos en tratados internacionales, entendiendo a ambas fuentes, como normas supremas de nuestro ordenamiento jurídico.

La tesis de referencia, señala que la supremacía a la que alude, "implica que los valores, principios y derechos que ellas materializan deben permear en todo el orden jurídico, obligando a todas las autoridades a su aplicación y, en aquellos casos en que sea procedente, a su interpretación", y que "en el supuesto de que un mismo derecho fundamental esté reconocido en esas dos fuentes supremas del ordenamiento jurídico (...), la elección de la norma que será aplicable -en materia de derechos humanos-, atenderá a criterios que favorezcan al individuo o lo que se ha denominado principio pro persona". Finalmente indica que "en caso de que exista una diferencia entre el alcance o la protección reconocida en las normas de estas distintas fuentes, deberá prevalecer aquélla que represente una mayor protección para la persona o que implique una menor restricción".

Estamos pues, frente a un nuevo modelo de instrumentación de la actividad jurisdiccional que imperativamente debe constituirse con su primer fundamento en los derechos humanos, ya no únicamente como un auténtica y legítima aspiración democrática, sino como un mandamiento constitucional ineludible.

\footnotetext{
${ }^{11}$ Al respecto es importante consultar la Contradicción de tesis 293/2011 entre las sustentadas por el primer Tribunal Colegiado en materias administrativa y de trabajo del Décimo Primer Circuito y el Séptimo Tribunal Colegiado en materia civil del Primer Circuito, pues estimamos que en dicha contradicción se establece una excepción al principio pro persona.

${ }^{12}$ Además este principio debe operar conjuntamente con los de universalidad, interdependencia, indivisibilidad y progresividad.
} 


\section{ALGUNAS NOTAS SOBRE LA ESPECIFICIDAD DE LA INTERPRETACIÓN CONSTITUCIONAL}

\subsection{CONSIDERACIONES INTRODUCTORIAS}

Estamos convencidos por lo dicho hasta aquí, que uno de los problemas emergentes más claros, por lo menos en su ubicación, derivado de la constitucionalización de los derechos humanos no solamente en el caso mexicano, sino en la teoría contemporánea del Derecho, es precisamente la interpretación de los mismos, y más puntualmente, su hermenéutica constitucional.

Una percepción podríamos decir, si no generalizada, considerable entre los juristas que se enfrentan a esta nueva realidad de los derechos humanos, es la vaguedad de sus contenidos, la dificultad de su aplicación, la asimilación correcta de la jerarquía de los diferentes órdenes normativos, y en suma, un espacio de incertidumbre que incluso pudiera originar contundencia en la seguridad jurídica.

Dicha percepción, si bien la comprendemos fundamentalmente en los actores jurídicos que cotidianamente tienen que aplicar la norma desde los tribunales, en las fiscalías o en las pretensiones de sus defendidos, pensamos no necesariamente debe ser motivo de desánimo, pesimismo u oposición categórica hacia el nuevo modelo, pues estimamos que el mismo, se encuentra en un proceso de construcción que posibilitará mejores condiciones para la efectividad de los derechos humanos desde una visión cada vez más integradora y por ello muy seguramente más efectiva.

\subsection{SOBRE LA INTERPRETACIÓN CONSTITUCIONAL}

La interpretación constitucional en México cobra aun mayor sentido en la asignatura de los derechos humanos, pues el monopolio de la SCJN y del Poder Judicial Federal en general, ha dejado de existir, abriéndose la puerta al control difuso de la constitucionalidad de los derechos humanos, lo cual hace más compleja, pero más rica y fundamentalmente pensamos más justa la cultura jurídica en nuestro sistema normativo. En este sentido, un tema toral es por supuesto el de la interpretación constitucional que a continuación desarrollaremos.

Los estudios sobre hermenéutica jurídica en general, no van de la mano, ni en tiempo ni en técnica de los estudios sobre la interpretación constitucional. De acuerdo con Carpizo, al respecto existen antecedentes y preocupaciones aislados, como las obras "Constitutional Limitations de Thomas Cooley (1868)", y "L'interpretazione delle leggi di diritto pubblico de Santi Romano (1899)"; y que en el siglo XX se ocuparon Smend, Miceli y Kelsen, pero es especialmente después de la segunda guerra mundial que se profundiza y amplía la preocupación por la interpretación constitucional como una consecuencia del nuevo constitucionalismo que aparece en las leyes fundamentales que los países promulgan después de la 
creación de cortes o tribunales constitucionales y del especial desenvolvimiento nacional e internacional de la protección de los derechos humanos ${ }^{13}$.

Pues bien, como se nota, no es una casualidad que el tema central de este texto resida en la interpretación constitucional de los derechos humanos, pues la conexión histórica establece un punto fundamental de partida para el desarrollo científico de la asignatura.

Igualmente es importante también situarnos en el significado del Estado Constitucional, pues este:

"se caracteriza en oposición al Estado de Derecho, por una decreciente densidad normativa constitucional, es decir, como manifiesta el profesor Werner Kagi, una Constitución de mínimos que entiende la división de poderes como cooperación natural y no obstrucción o indiferencia de unos poderes sobre otros" (...), "en los Estados Constitucionales existe un declive claro y contundente de la densidad normativa (disminución de los preceptos fundamentales constitucionales), y por otro lado, una disminución de la firmeza y precisión de las normas (inclinación a debilitar y cuestionar su sentido jurídico normativo), es decir, existe la búsqueda constante de un constitucionalismo interpretativo abierto que impregna y dirige todo el ordenamiento jurídico con la finalidad de garantizar el contenido esencial de los derechos fundamentales". ${ }^{4}$

Aguilera Portales, al referirse además al Estado Constitucional, señala la importancia en el fortalecimiento del poder judicial como órgano de control y cooperación del legislativo. También por supuesto que nos llama la atención de la importancia de la garantía de los derechos fundamentales que imprime el Estado Constitucional, que sigue fortaleciendo esa idea "no casual" de surgimiento del mismo como lo ha señalado Ferrajoli, en el momento de proliferación de la internacionalización de los derechos humanos, así pues el constitucionalismo, como nuevo paradigma jurídico diferente al positivismo clásico, sugiere la incorporación de valores al texto constitucional, los cuales permiten una interpretación más abierta e incluso la adaptación del texto constitucional ${ }^{15}$.

\subsubsection{HACIA UNA CONCEPTUALIZACIÓN DE LA INTERPRETACIÓN CONSTITUCIONAL}

Una interrogante absolutamente necesaria al plantearnos la idea de una conceptualización de la interpretación constitucional, es si ésta es o debe ser diferente a la interpretación de la ley en términos generales. En este tenor, en principio, Guastini afirma que muchas de las características que concurren en los textos constitucionales, no son propias o exclusivas de estos, sino que también puede predicarse de muchas otras normas que integran el ordenamiento jurídico; y que así la importante presencia de principios en las Constituciones tam-

${ }^{13}$ CARPIZO, Jorge (Prólogo), en CARMONA TINOCO, Jorge Ulises, La interpretación judicial constitucional, $1^{\text {a }}$ ed., IIJUNAM-CNDH, México, 1996, p. 9.

${ }^{14}$ AGUILERA PORTALES, Rafael Enrique, "Estado Constitucional, derechos fundamentales e interpretación constitucional", en http://biblio.juridicas.unam.mx/libros/6/299o/3.pdf, fecha de consulta: 10 de abril de 2013, p. 27.

${ }^{15}$ DE MONTALVO JÄÄSKELÄINEN, Federico, "La interpretación constitucional: problemas de método e intérprete" en Icade, Revista Cuatrimestral de las Facultades de Derecho y Ciencias Económicas y Empresariales, N. 82, enero-abril, Universidad Pontificia Comillas, España, 2011, p. 57. 
bién puede producirse en cualquier ley, ${ }^{16}$ no obstante el jurista en otra obra que ya hemos citado establece una idea sobre la especificidad en la interpretación constitucional que más adelante comentaremos. Por ahora, ya que hemos referido el tema de los principios, es importante hacer mención de la consideración de Montalvo Jääskeläinen, cuando señala que:

"si puede afirmarse que la interpretación de las normas constitucionales responde a ciertos principios singulares que si bien, puede recurrirse a ellos en ocasiones por el intérprete de la ley, ello no es siempre así. Las características que siempre han de concurrir en la norma constitucional y que no necesariamente tienen que concurrir en la norma legal informan a favor de la existencia de unos principios singulares de interpretación constitucional". ${ }^{17}$

En este tenor, De Montalvo, establece como principios específicos el de "interpretación conforme", y el de "conservación de la norma", el primero que determina que el juzgador ha de elegir entre la interpretación más conforme con lo dispuesto en la norma jerárquicamente superior; y el segundo que se refiere a que supone que "el operador jurídico, fundamentalmente, el juzgador ha de optar por la constitucionalidad de la ley en aquellos casos en los que existan dudas acerca de su validez". ${ }^{18}$ De las consideraciones anteriores, vamos caminando hacia la especificidad de la interpretación constitucional en el sentido de la identificación de "principios igualmente específicos", que nos recuerdan desde ahora, el "pro persona", de indivisibilidad, progresividad y universalidad, entre otros, que precisamente son lo que refiere el nuevo orden constitucional de los derechos humanos.

Pero otra de las características particulares de la interpretación constitucional es precisamente su punto de partida, pues como lo establece el jurista que venimos citando, ese punto no es filosófico, sino político,

"de manera que ésta aspira a evitar conflictos mediante la adopción de soluciones que satisfagan a todos en el mayor grado que las circunstancias permitan"19, pues "la gran trascendencia política de muchos de los preceptos de la Constitución, obliga no sólo plantearse la validez de la norma, sino también las consecuencias de su aplicación. Sin embargo, ello no significa que haya que ceder el enfoque propio del derecho al de la Política, sino razonar jurídicamente sobre las consecuencias políticas de los preceptos constitucionales."20

Como podemos verificar, nos encontramos ante una asignatura compleja y que puede ser abordada bajo varias perspectivas, no obstante es importante desde ahora acercarnos a una conceptualización de la interpretación constitucional, antes de entrar al tema de su especificidad. En este orden de ideas de acuerdo con Arteaga Nava, esta interpretación es en fondo y forma una parte de la interpretación jurídica, aunque la interpretación constitucional debe hacerse con base en ciertos principios especiales, lo cual no quiere decir que se trate de una forma independiente, especial o diferente de interpretación jurídica ${ }^{21}$. De lo anterior, estimamos que si como lo afirma el autor estamos frente a una "parte de la investigación

\footnotetext{
${ }^{16}$ GUASTINI, Ricardo, Teoría e Ideología de la Interpretación Constitucional, $1^{\mathrm{a}}$ ed., Trotta, Madrid, 20o8, pp. 54-58.

${ }^{17}$ DE MONTALVO JÄÄSKELÄINEN, Federico, Op. Cit., p. 57.

${ }^{18}$ Idem.

${ }^{19}$ Ibidem., p. 53.

${ }^{20}$ Idem.

${ }^{21}$ ARTEAGA NAVA, Elizur, Derecho Constitucional, $3^{\text {a }}$ ed., Oxford, México, 2008, p. 40. 
jurídica”, se admite una cierta autonomía en su conceptualización, que si bien pertenece al género de la hermenéutica jurídica, si es susceptible de estudiarla en particular. Interpretar la Constitución,

“es comprender el sentido de un precepto con base en sí mismo, en los términos en que está redactado y en todo su contexto. El objeto integral de la interpretación constitucional o legal es poner en práctica la interpretación de los autores del documento $\mathrm{y}$, adicionalmente, la intención del pueblo al adoptarlo. Este propósito conjuntamente con el fin general de cada disposición particular, debe, por consiguiente, ser tenido en vista constantemente". ${ }^{22}$

Hasta aquí, al parecer aún no tenemos claridad en la conceptualización de la interpretación constitucional, a pesar de que la referencia a los principios es contundente, por ello, nos sirven las reflexiones de Guastini sobre el particular, cuando establece algunas variables que hay que tener en cuenta en la interpretación del texto constitucional y que citamos a continuación ${ }^{23}$ :

Dos concepciones de la Constitución (que limita el poder político, o bien la función de modelar las relaciones sociales).

a) El régimen jurídico de la Constitución (Constituciones rígidas o flexibles), y

b) El contenido normativo de la Constitución, en donde el autor refiere que hay Constituciones que contienen exclusivamente normas sobre organización de los poderes o bien incluyendo además un catálogo de derechos.

Teniendo en cuenta esta estructura, por supuesto estimamos que la especificidad de la interpretación constitucional en el caso mexicano, está en el contenido normativo de la Constitución, con la interpretación de los derechos y los principios.

Ahora bien, siguiendo con el estudio del jurista, éste sin ser exhaustivo, señala algunos problemas de la interpretación constitucional que pensamos abonan a la idea de establecer una noción sobre dicha actividad hermenéutica, por lo que los sintetizamos a continuación: ${ }^{24}$

- Problemas en las Constituciones que no disponen sobre su reforma.

- El dilema del reconocimiento o la creación de derechos.

- La pregunta de si los preámbulos expresan normas.

- La cuestión de si existen límites lógicos a la reforma (por ejemplo una cosa sería la reforma de la Constitución, y otra la instauración de una nueva Constitución, igualmente se dice que en ningún caso la reforma puede modificar los principios superiores de la Constitución.

\footnotetext{
${ }^{22}$ Idem., p. 44.

${ }^{23}$ GUASTINI, Ricardo, Estudios sobre la interpretación jurídica, (Gascón, Marina y Carbonell, Miguel, Trad.),

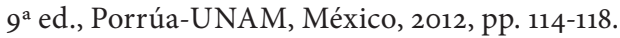

${ }^{24}$ Idem., pp. 123-131.
} 
- La plenitud de la Constitución; si la misma es completa, para cualquier ley existirá un parámetro de legitimidad constitucional; pero si es incompleta, existirán leyes que no podrán ser confrontadas con alguna norma constitucional para valorar su conformidad con la Constitución.

Por último, explica Guastini que para algunos la plenitud de algunas Constituciones "es sostenible a partir del principio de igualdad; especialmente, si se entiende como una prohibición general de discriminación (dirigida al legislador), o incluso como un principio de razonabilidad de la legislación (según la práctica de algunos tribunales constitucionales)". ${ }^{25}$

\subsubsection{LA ESPECIFICIDAD DE LA INTERPRETACIÓN CONSTITUCIONAL}

En primer término, como lo hemos ido delimitando, estimamos que efectivamente si podemos referirnos a una especificidad de la interpretación constitucional, el problema más complejo ahora, será definir sus elementos. En este sentido, es importante partir de una consideración evidente: en principio lo que hace diferente a la interpretación constitucional, es precisamente su objeto, es decir la Constitución, y este primer rasgo de la especificidad está relacionado "tanto en su carácter abierto, lo que se traduce en una mayor presencia en la misma de valores, principios y conceptos jurídicos indeterminados, como por su carácter eminentemente político". ${ }^{26}$

La función de la norma constitucional, no está orientada a la solución de casos concretos, sino fundamentalmente a la organización del Estado y al establecimiento de derechos, por ello plantea el autor, que existen diferencias respecto a la interpretación de la ley y de la Constitución, pero no en los métodos, sino en el objeto. ${ }^{27}$

Respecto al contenido, en su mayor parte indeterminado de la Constitución, precisamente Häberle sitúa el punto de partida de su doctrina en dicho contenido, por consiguiente señala que "la Constitución vive de la interpretación", ${ }^{28}$ afirmación que dota de aun más importancia a la delimitación de dicha actividad hermenéutica.

El mismo autor distingue entre interpretación e intérpretes en sentido amplio y en sentido estricto, en este último sentido, es una actividad consciente dirigida a la comprensión y aplicación de la norma. La interpretación en sentido amplio, comprende cualquier actualización de la Constitución. ${ }^{29}$ La puesta en práctica de la Constitución, es pues la interpretación de la misma en sentido amplio, y en ella participan todos los ciudadanos, grupos sociales y órganos estatales, todos ellos, fuerzas productivas interpretadoras que proporcionan materiales hermenéuticos a los intérpretes en sentido estricto, por lo que culmina el autor señalando que la interpretación de la que vive la Constitución, es una forma de participación cívica y democrática. ${ }^{30}$ Dworkin, estimamos es coincidente con dicho proceso entendido fundamentalmente en sentido amplio, cuando indica que la interpretación de la Constitución no

\footnotetext{
${ }^{25}$ Ibidem., p. 131.

${ }^{26}$ DE MONTALVO JÄÄSKELÄINEN, Federico, Op. Cit., p. 45.

${ }^{27}$ Idem., p. 48.

${ }^{28}$ AGUILERA PORTALES, Rafael Enrique, Op. Cit., p. 30.

${ }^{29}$ Ibidem.

30 Idem.
} 
corresponde únicamente a las instancias estatales, incluso ni a los órganos jurisdiccionales, ensanchando la concepción de democracia jurisdiccional. ${ }^{31}$

Regresando al tema de los principios que hemos tratado en el apartado anterior, es importante verificar que "la regla es susceptible de ser interpretada como paso previo a su aplicación en la resolución del conflicto concreto y el principio, por el contrario, requiere ser interpretado para, a su vez, servir de herramienta para la interpretación de la norma", ${ }^{2}$ por lo que la interpretación de los principios es tan importante, que sin ese desarrollo, no puede ser óptimamente resuelto un caso concreto, por carecer del presupuesto interpretativo fundamental (contenido del principio).

De lo analizado hasta ahora, pensamos que entonces uno de las tareas para la investigación jurídica en los tópicos que tratamos en este trabajo (interpretación constitucional y derechos humanos) es precisamente la demarcación de principios de los cuales Artega Nava señala algunos ${ }^{33}$ enfatizando que éstos delimitan la especificidad constitucional, a los que por supuesto en sintonía con el artículo primero constitucional, habrá que agregar los siguientes específicamente en tratándose de derechos humanos: pro persona, universalidad, interdependencia, indivisibilidad y progresividad.

Pues bien, evidentemente no hemos sido exhaustivos en el estudio de la interpretación constitucional, pues por ejemplo, faltarían entre muchas otras cuestiones, revisar el denominado por Susana Pozzolo Neoconstitucionalismo, que constituye el conjunto de posiciones dogmáticas que agrupan diversos autores, ${ }^{34}$ precisamente respecto de temas como la interpretación constitucional y los derechos fundamentales, pues como señala la jurista en comento, "la tesis sobre la especificidad de la interpretación constitucional encuentra partidarios en diversas disciplinas, en el ámbito de la filosofía del derecho viene defendida, en particular, por un grupo de iusfilósofos que comparten un peculiar modo de acercarse al derecho".35

La interpretación constitucional de los derechos humanos, se nos presenta entonces como un tema ineludible en el paradigma constitucional que vivimos y cuyas consecuencias se encuentran en construcción. Las herramientas del derecho constitucional y fundamentalmente del derecho procesal constitucional deberán entonces adaptarse a estos nuevos tiempos donde la efectividad normativa de los derechos, sigue estando en buena medida pendiente.

\section{REFLEXIONES FINALES}

Hay por lo menos dos temas puestos en la mesa de la discusión académica y jurisdiccional con absoluta vigencia en México desde el año 2011, fecha en la que se reformó la Constitución en materia de Derechos Humanos de manera sustancial, nos referimos, por un lado al

\footnotetext{
${ }^{31}$ Ibidem., p. 34 .

32 DE MONTALVO JÄÄSKELÄINEN, Federico, Op. Cit., p. 55.

${ }^{33}$ Por ejemplo: Supremacía constitucional, de que la Constitución es norma fundamental, la Constitución es una y completa en sí y por sí, de incongruencia inconstitucional, de permanencia constitucional, Generalidad y Exclusividad, que regula la existencia de derechos humanos y sus limitantes, del silencio de la constitución, de que todo poder es completo en sí mismo, de que la Constitución es un texto político, que regulan la interpretación de las prohibiciones constitucionales, de Jerarquía, etc.

34 Como DWORKIN, Alexy, ZAGREBELSKY y en parte SANTIAGO Nino.

35 POZZOLO, Susana, Neoconstitucionalismo y especificidad de la interpretación constitucional, en línea: http://www.cervantesvirtual.com/obra/neoconstitucionalismo-y-especificidad-de-la-interpretacin-o/, fecha de consulta: 15 de Febrero de 2013.
} 
nuevo modelo constitucional construido a la luz de los estándares del derecho internacional de los derechos humanos, y por otra parte a la renovada forma que debe adquirir la interpretación constitucional de dichos derechos.

Resulta normal, como ocurre con el advenimiento de cualquier otro paradigma jurídico, que la incertidumbre de las y los juristas sea un lugar común en este momento histórico determinado por la importancia del diseño constitucional de los derechos, y fundamentalmente en la preocupación por su efectividad. La interpretación constitucional debe, ineludiblemente observarse desde la perspectiva de los estándares nacionales e internacionales de los derechos humanos, por lo que la congruencia entre los diversos planos normativos, será fundamental en la consolidación del nuevo orden constitucional.

BIBLIOGRAFÍA

AGUILERA PORTALES, Rafael Enrique, "Estado Constitucional, derechos fundamentales e interpretación constitucional", en http://biblio.juridicas.unam.mx/libros/6/299o/3. pdf, fecha de consulta: 10 de abril de 2013.

ARTEAGA NAVA, Elizur, Derecho Constitucional, $3^{\text {a }}$ ed., Oxford, México, 2008.

CARMONA TINOCO, Jorge Ulises, La interpretación judicial constitucional, $1^{\mathrm{a}}$ ed., IIJUNAM-CNDH, México, 1996.

DE LA TORRE TORRES Rosa María, "Ponencia: El nuevo paradigma de los derechos humanos en México", V Congreso Nacional de Derecho Constitucional, UNAM, México, D.F., Octubre de 2012.

DE MONTALVO JÄÄSKELÄINEN, Federico, "La interpretación constitucional: problemas de método e intérprete" en Icade, Revista Cuatrimestral de las Facultades de Derecho y Ciencias Económicas y Empresariales, N. 82, enero-abril, Universidad Pontificia Comillas, España, 2011.

FERRER MAC GREGOR, Eduardo, “Interpretación Conforme y Control Difuso de Convencionalidad: El nuevo paradigma para el Juez Mexicano", en Estudios Constitucionales, Centro de Estudios Constitucionales de Chile Universidad de Talca, Año 9, No. 2, 2011.

GUASTINI, Ricardo, Teoría e Ideología de la Interpretación Constitucional, $1^{\mathrm{a}}$ ed., Trotta, Madrid, 2008.

, Estudios sobre la interpretación jurídica, (Gascón, Marina y Carbonell, Miguel, Trad.), 9ª ed., Porrúa-UNAM, México, 2012.

LARA PONTE, Rodolfo, Los Derechos Sociales en el Constitucionalismo Mexicano, $4^{\mathrm{a}}$ ed., Porrúa, México, 2007.

SÁNCHEZ CORDERO, Olga, La interpretación de los derechos fundamentales por la Suprema Corte de Justicia de México, en línea: http://www.scjn.gob.mx/conocelacorte/ ministra/o1junio-conferencia-derechos-fundamentales.pdf, fecha de consulta: 1 de enero de 2013. 
POZZOLO, Susana, Neoconstitucionalismo y especificidad de la interpretación constitucional, en línea: http://www.cervantesvirtual.com/obra/neoconstitucionalismo-y-especificidad-de-la-interpretacin-o/, fecha de consulta: 15 de Febrero de 2013.

VIDAURRI ARÉCHIGA, Manuel, SORIANO FLORES José Jesús, “El Contenido de la Reforma Constitucional de 2011 en materia de Derechos Humanos: 10 Temas Fundamentales", en Revista Penal, Núm. 30, Universidad de Huelva, España, 2012. 